\title{
2D Surface Spin Waves in Dynamic Magnonic Crystals Created by a Surface Acoustic Wave in YIG Films
}

\author{
Alexander V. Medved \\ Fryazino Branch, Kotel'nikov Institute of Radioengineering and Electronics, Russian Academy of Sciences, Fryazino, Russia \\ Email address: \\ avm@ms.ire.rssi.ru

\section{To cite this article:} \\ Alexander V. Medved. 2D Surface Spin Waves in Dynamic Magnonic Crystals Created by a Surface Acoustic Wave in YIG Films. American \\ Journal of Physics and Applications. Vol. 9, No. 5, 2021, pp. 110-115. doi: 10.11648/j.ajpa.20210905.12
}

Received: August 16, 2021; Accepted: September 16, 2021; Published: September 27, 2021

\begin{abstract}
Some results of the study of 2D propagation of surface magnetostatic spin waves (SMSW) in dynamic magnonic crystals (DMC) created by a surface acoustic wave (SAW) in a structure with a yttrium-iron garnet (YIG) film are presented. Such studies are interesting from the practical point of view of creating real relatively complex devices on spin waves when they propagate in different directions, and not only in the 1D case. The methods of experimental research are presented, in particular, the features of the method of excitation of SAW in the structure that do not lead to the appearance of additional magnetic anisotropy in it, a method for measuring the angular dependencies of SMSW using mobile antenna-probes is presented. The angular dependences of the magnonic band gap frequencies are measured. It is established that the transmission bands with the transformation of the reflected SMSW into other types of magnetostatic waves (MSW) exist at any angle values, while the intervals in which there are no SMSW transformations during reflections occur in a certain narrower range of angles. The angles of the directions of the wave vectors and the Poynting vector of the reflected SMSW were also measured. A satisfactory agreement was obtained with the calculation performed using the method of isofrequency curves and the laws of inelastic scattering SMSW on SAW.
\end{abstract}

Keywords: 2D Spin Wave Propagation, Dynamic Magnonic Crystal, Yttrium Iron Garnet, ZnO Film, Surface Acoustic Wave, Mobile Probe-antenna, Cubic Magnetic Anisotropy, Izofrequency Curves

\section{Introduction}

The «magnonic crystals», by analogy with photonic crystals, denote a magnetic medium with artificially created periodic magnetic inhomogeneities [1-3]. Spin waves propagating in such a medium (including surface magnetostatic waves) undergo Bragg reflection at certain frequencies. For these waves, the so-called magnonic forbidden bands (magnonic gaps) arise at these frequencies. Spin waves at the frequencies of these forbidden bands cannot propagate and experience reflections. The concept of magnonic crystals is widely used at present in the study of magnetic phenomena in garnet ferrite. Some signal processing devices have been proposed, the principle of operation of which is based on this concept (see for example [4-9]).

Surface acoustic waves (SAW) were used to create magnetic inhomogeneities in YIG films with surface magnetostatic (spin) waves (SMSW) propagating in them [10]. These inhomogeneities change in time and space in a wave-like manner determined by the propagating acoustic wave (SAW). Thus, such a magnonic crystal is a dynamic magnonic crystal (DMC). The physical phenomenon underlying such DMCs is the inelastic scattering of spin waves on an acoustic wave [11-17]. Such DMC have been used to measure the parameters of surface magnetostatic waves and bulk magnetostatic waves caused by magnetic anisotropy in YIG films [18, 19]. Several devices for processing microwave signals $[20,21]$ have been proposed and experimentally demonstrated, the principle of operation of which is based on the properties of SAW-DMCs, including their special non-reciprocity properties [22].

In all these applications, the one-dimensional geometry of DMC was exploited, SMSW and SAW mainly propagated collinearly. In this paper, we consider a two-dimensional geometry with SMSW and SAW propagating at different angles to each other in DMC with the structure "yttrium-iron garnet (YIG) film on a gallium-gadolinium garnet (GGG) substrate". The method of experimental research is described. The results of measuring the bandgap frequency for SMSW 
propagation as a function of the angles between the SMSW and SAW wave vectors in the DMC in the plane of the YIGGGG structure are presented. The results of measuring the directions of the wave vectors of the reflected SMSW and the energy flow vectors at the bandgap frequencies are also presented. The experimental results obtained are compared with the corresponding theoretical dependences obtained graphically using the method of isofrequency curves [23], also known in geometric optics, and the previously developed theory of inelastic scattering of SMSW on acoustic waves [12-17] - the phenomenon underlying the operation of dynamic magnonic crystals created by propagating acoustic waves in magnetic materials. Satisfactory agreement of the compared results was obtained.

The results described in our article can be used in the development of new 2-D spin-waves devices and the study of 2-D spin wave propagation processes.

\section{Experimental Technique}

The experimental study of the 2-D SMSW propagation was carried out in the SAW-DMC, created in YIG-GGG structures in the form of plates (wafers) with a diameter of $60 \mathrm{~mm}$ and a crystallographic axis $<111>$ perpendicular to the plate plane. The monolithic configuration of the SAW-YIG magnonic crystal was used, as it was called in [10], a piezoelectric $\mathrm{ZnO}$ film with interdigital transducers (IDT) deposited on the surface of the YIG film was used to excite the SAW. The resonant frequency of these IDTs was $41 \mathrm{MHz}$. The deposition of $\mathrm{ZnO}$ films on the surface of the YIG-GGG structure leads to the appearance of a significant uniaxial magnetic anisotropy in the YIG film (see [19, 24]). Therefore, in order not to complicate the interpretation of the experimental results, $\mathrm{ZnO}$ films with IDT were deposited through a special mask only at the edges of the SAW channels, at their beginning and end, as shown in Figure 1a and Figure 1b. These Figures show schematically the configurations of the SAW-YIG DMC samples and the schemes for experimental investigating of wave propagations in these samples. The thicknesses of the
$\mathrm{ZnO}$ films were 5 microns, and the distance between the IDTs in the channels was $30 \mathrm{~mm}$. The apertures of IDTs were $5 \mathrm{~mm}$. The insertion loss of SAW channels (with matching inductors) was no more than $20 \mathrm{~dB}$. Microwave mobile antenna- probes were used to excite and receive SMSW $[25,26]$. These probes could move across the entire working surface of the structures and rotate in the X-Y plane (see Figure 1). The actual antennas in the probes were wire conductors with a thickness of 10 microns and an aperture of $4 \mathrm{~mm}$. The antennas were pressed against the free surface of the YIG film.

The studied SAW-YIG DMC samples were placed in the tangential magnetic field of a permanent magnet. The dependences of the bandgap frequency and the direction of the wave vectors of the reflected SMSW on the angle between the incident SMSW and SAW were measured. The measurements were carried out in the pulse mode of excitations of SMSW and SAW, which made it possible to more accurately notice the change in the level of the passing SMSW when the frequencies of the incident SMSW hit the forbidden magnonic gap. The duration of the SAW pulses was 2-5 microseconds, and the SMSW pulses were 15-20 microseconds. The pulse repetition rate was $200 \mathrm{~Hz}$. The microwave pulses exciting the SAW and the SMSW were synchronized in a certain way so that the SAW pulse passed in the sample when the SMSW also propagated in it.

The configuration of the samples used and the experimental schemes are shown in Figure 1 a) and $1 \mathrm{~b}$ ). The envelopes of the microwave signals supplied and removed from the corresponding antennas and IDTs are also schematically shown in Figure 1. Figure 1a) illustrates the measurements of the band gap frequencies by the "on-pass" method. In this case, the antennas " 1 " and " 2 " were located parallel to each other and to the direction of the magnetic field, as shown in Figure 1 a). The values of the frequency $f_{i}$ ere measured at which the envelope of the SMSW signal taken from the output antenna "2" had the maximum depth of the pit caused by the SMSW falling into the forbidden magnonic band created by SAW pulses.
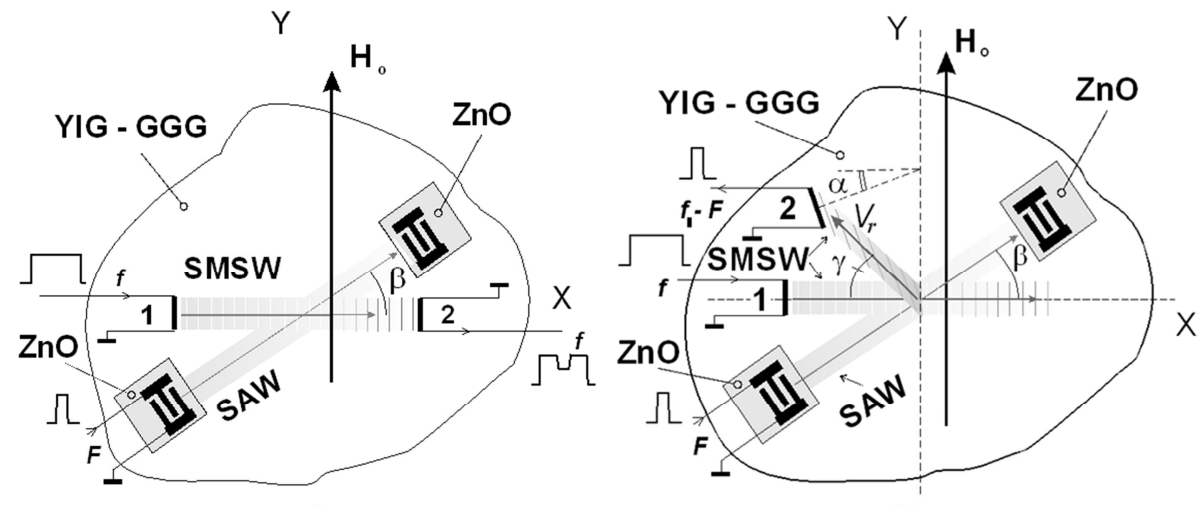

b)

Figure 1. SAW-DMC samples configurations and experimental schemes: a) the "on pass" method, b) the "on reflection" method. 1 and 2 - mobile probesantennas.

Figure $1 \mathrm{~b}$ ) shows the scheme of the measurements of the bandgap frequencies by the "on-reflection" method. In contrast to the one-dimensional case, in the two-dimensional case where the SMSW propagates in the DMC, the wave vectors of 
the reflected SMSW (of the frequency $f_{i}-F$ ) will not necessarily lie on a straight line with the wave vector of the incident wave. Moreover, the direction of the wave vector of the reflected wave does not necessarily coincide with the direction of its Poynting vector. Therefore, in the experiment, the output mobile antenna "2" from the position shown in Figure 1 a) was moved to a position on the working surface of the structure at which the signal of the frequency $\left(f_{i}-F\right)$ taken from this antenna would be the maximum. The value of this frequency, $f_{i}$, was measured. The input mobile antenna "1" in this case, as in the experiments "on-pass", was adjusted parallel to the external magnetic field. The angles of rotation.

$\alpha$, and the angle $\gamma$ of the direction of the antenna "2" were also measured, which made it possible to determine the direction of the wave vector and the direction of the energy flow of the reflected wave in the SAW-DMC.

All these measurements were performed as functions of the angle $\beta$ between the wave vectors of the incident SMSW and SAW. The angle $\beta$ can be changed by rotating the structure understudy in the X-Y plane, while leaving the mobile antennas "1" and "2" in the "on-pass" method and the antenna "1" in the "on-reflection" method parallel to the Yaxis (the direction of the external magnetic field).

The power of the SAW used in our experiments was about 50 $\mathrm{mW}$. The level of the microwave signals applied to the input mobile antennas to excite the SMSW did not exceed the value at which nonlinear effects began to appear in the DMC [27].

\section{Experimental Results and Discussion}

In our experiments with DMC in the "on-pass" method, at the angle between the wave vectors of the incident SMSW and SAW $\beta=0$ (see Figure 1a), an essentially onedimensional geometry is realized, which was studied in our previous works (see for exampl [10]). Several magnonic gaps for SMSW were observed in our experiments as in [28]. One magnonic band gap occurs at the highest frequencies of the SMSW spectrum. It is denoted as an "ordinary" one and described by the theory of inelastic scattering of SMSW by SAW. The remaining bandgaps, if any, lie in frequency closer to the beginning of the spectrum and arise due to scattering with the transformation of the SMSW at the reflection into bulk modes of the so-called anisotropic-dipole magnetostatic waves (ADMSW) [15, 19, 28]. Following the terminology used in [19], we will refer to these gaps as additional ("extra") gaps. When the angle $\beta$ was increased to certain limits, this situation persisted.

The magnonic band gaps frequencies were measured as functions of the angle $\beta$. Figure 2 shows the results of the measurement for a DMC sample with a YIG film thickness $\mathrm{d}=9.4$ microns in a magnetic field of $\mathrm{H}_{\mathrm{O}}=640$ Oe (hollow square dots). The antennas "1" and "2" were placed parallel to each other and parallel to the external magnetic field, the distance between the antennas was $20 \mathrm{~mm}$. When measuring the frequencies of extra forbidden gaps, a situation arises when it is impossible to resolve individual gaps that are close in frequency to each other, and then the highest and lowest frequencies were recorded in the experiment (in Figure 2 open round dots and filled round dots, respectively). Thus, the boundaries of the frequency ranges in which these magnonic gaps exist at a given angle value $\beta$ were measured. Such frequency ranges are represented by the shaded areas in Figure 2.

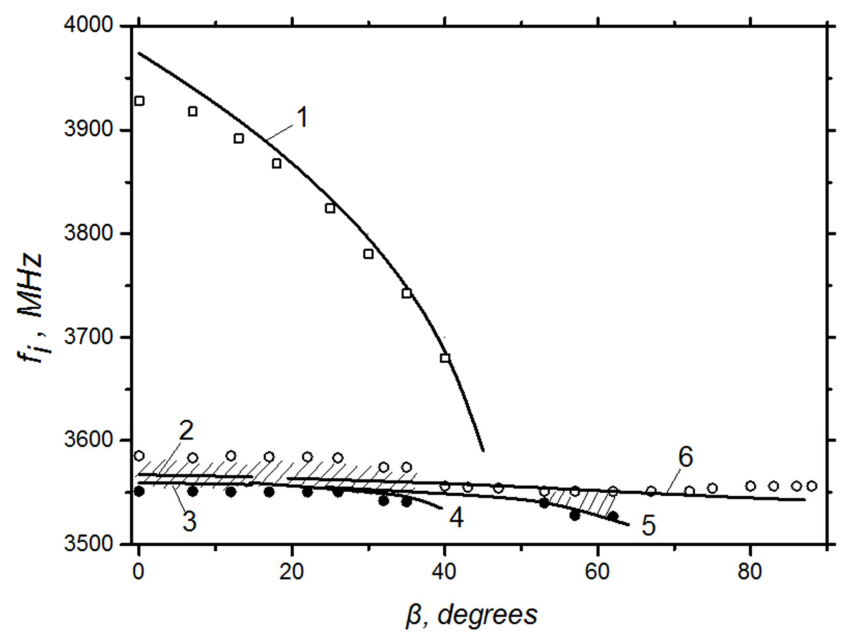

Figure 2. The magnonic band gap frequencies fi as functions of the angle $\beta$ between the wave vectors of incident SMSW and SAW in DMC. Dotsmeasurements by "on pass" method. Solid lines - calculations: 1 "ordinary" magnonic gap, 2 and 3 are for "extra" gaps with the reflections with transformations to 1-st and 2-nd modes of ADMSW, 4- "extra" gaps with transformation to zero mode of the BVMSW, 5 - "extra" gap with transformations to the first mode of the BVMSW, and curve 6-to the second mode. Shaded zones show areas where it is difficult to distinguish individual gaps in experiments. $H_{O}=640 \mathrm{Oe} . d=9.4 \mathrm{mcm} . \mathrm{F}=41 \mathrm{MHz}$.

It can be seen that the ordinary magnonic gap was observed up to the values of the angles $\beta$ of $\approx 40$ degrees, while the extra gaps were observed at all values of this angle.

Figure 3 shows the results of measuring and calculating the dependences of the frequency of the "ordinary" magnonic band gap and the angle of the direction of the wave vector of the reflected SMSW as a function of the angle between the directions of the incident SMSW and the SAW for a sample with a YIG film with a thickness of 5.7 microns in a magnetic field of 595 Oe. The same Figure shows also the results of measuring and calculating the direction of the Pointing vector (angle $\gamma$ ) for the reflected SMSW. These measurements were carried out by the "on reflection" method (see Figure 1a). The "ordinary" magnonic bandgap was observed, as in the Figure 2, up to $\approx 40$ degrees angles between the incident SMSW and SAW.

The solid curves in Figure 2 and Figure 3 represent the results of the calculation of the corresponding dependences, made graphically from the calculated isofrequencies of the propagating MSW and the laws of inelastic scattering in $k_{X}-k_{Y}$ coordinates with the parameters of YIG films and the magnetic field corresponding to the experiment.

The initial equations for calculating the isofrequency curves are the equations of magnetostatic and the motion of the magnetization vector [29] with using magnetic and thermodynamic potentials. The thermodynamic potential $\Phi$ for the plane (111) of the film of a cubic ferromagnet, 
without taking into account the uniaxial induced anisotropy, in polar coordinates, has the form [30]:

$$
\begin{gathered}
\Phi=-\mathrm{MH} \sin \theta \cos \left(\xi-\xi_{H}\right)-\left|K_{1}\right|\left[\frac{1}{3} \cos ^{4} \theta+\right. \\
\left.\frac{1}{4} \sin ^{4} \theta-\frac{\sqrt{2}}{3} \cos \theta \sin ^{3} \theta \sin 3 \xi\right]+\Phi_{M C}
\end{gathered}
$$

Here $\mathrm{H}$ is the external magnetic field, $\mathrm{M}$ is the magnetization, $\theta$ and $\xi$ are the polar and azimuthal

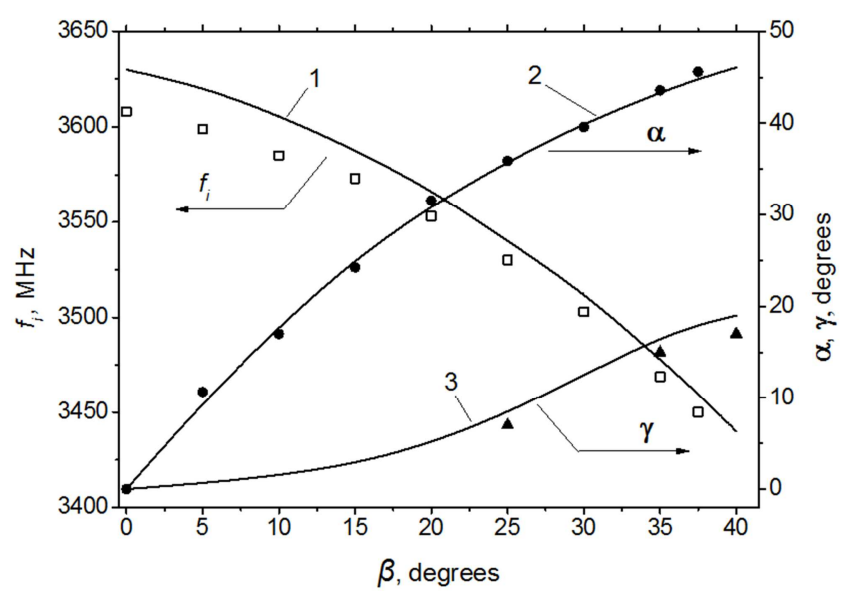

Figure 3. Frequency of the "ordinary" magnonic band gap (1), the angle of the direction of the wave vector of the reflected SMSW, $\alpha,(2)$ and the angle of the direction of the Pointing vector, $\gamma$, (3) for the reflected SMSW as functions of the angle between the directions of the incident SMSW and the $S A W, \beta$, for a sample with a YIG film with a thickness of 5.7 microns in a magnetic field of 595 Oe. The "on reflection" method used. (See also Figure 4) angles of the magnetic moment counted down from the crystallographic axes $\left\langle 111>\right.$ and $\langle\overline{1} 10\rangle$, respectively, $\xi_{H}$ is the direction of the external magnetic field, $\Phi_{M C}$ is the energy of the magnetostatic interaction.

The linearized equations of magnetostatics and motion of the magnetization vector are solved with boundary conditions

$$
\left.\Psi\right|_{z= \pm} d / 2=\text { const, } 4 \pi M_{z}+\left.\frac{\partial \Psi}{\partial z}\right|_{z= \pm} d / 2=\text { const, }
$$

where $d$ is the film thickness, the $z$ axis is parallel to the direction $<111>$, $\psi$ is the magnetic potential.

The solution is simplified if $\mathrm{H}$ coincides with one of the crystallographic directions $\{110\}\left(\xi_{H}=2 \pi n / 6, n=0,1,2 \ldots\right)$. For the sake of certainty, assume that $\xi_{H}=0$. Our estimates show that this assumption leads to an additional error in determining the angular dependences of the magnonic gap frequencies of no more than $5 \mathrm{MHz}$. The equilibrium direction of the magnetization $\theta_{0}, \xi_{0}$ is determined from the conditions of the minimum thermodynamic potential $\Phi$, when $\Phi_{M C}=-2 \pi M^{2} \sin ^{2} \theta$

$$
\frac{\partial \Phi}{\partial \theta}=0, \frac{\partial \Phi}{\partial \xi}=0
$$

It follows from this that $\xi_{0}=\xi_{H}=0$

$$
\begin{gathered}
-M H \cos \theta_{0}-\left|K_{1}\right|\left[-\frac{4}{3} \cos ^{3} \theta_{0} \sin \theta_{0}+\sin ^{3} \theta_{0} \cos \theta_{0}+\right. \\
\left.\frac{\sqrt{2}}{3} \sin ^{4} \theta_{0}-\sqrt{2} \sin ^{2} \theta_{0} \cos ^{2} \theta_{0}\right]-4 \pi M^{2} \cos \theta_{0} \sin \theta_{0}=0
\end{gathered}
$$

The equations of the dispersion characteristics of MSW propagating in the film are derived from the solvability conditions linearized near the equilibrium positions $\left(\theta_{0}, \xi_{0}\right)$ (see (3), (4)) and taking into account the boundary conditions (2) of the initial equations of magnetostatics and the motion of the magnetization vector. Omitting the intermediate calculations that are standard for linear boundary problems on eigenvalues, the final result is the following [13].

For SMSW

$$
\begin{gathered}
q^{2}\left(1+\chi_{2}\right)^{2}+\left(k_{X}^{2}+k_{Y}^{2}-\chi^{2} k_{Y}\right)+2 \sqrt{k_{X}^{2}+k_{Y}^{2}} q(1+ \\
\left.\chi_{2}\right) \operatorname{coth}(q d)=0
\end{gathered}
$$

where

$$
\begin{gathered}
q^{2}=\frac{k_{X}^{2} \chi_{2} \cot ^{2} \theta_{0}-\left(1+\chi_{2}\right)\left[k_{X}^{2}\left(1+\chi_{2} \cot ^{2} \theta_{0}\right)+k_{Y}^{2}\left(1+\chi_{1}\right)\right]}{\left(1+\chi_{2}\right)^{2}}>0 \\
\chi_{1}=\frac{\omega_{M}\left(\gamma \frac{\Phi_{\theta \theta}}{M}-\omega_{M} \cos ^{2} \theta_{0}\right)}{\omega_{0}^{2}-\omega^{2}}, \chi_{2}=\frac{\omega_{M} \gamma \frac{\Phi_{\xi \xi}}{M}}{\omega_{0}^{2}-\omega^{2}} \\
\chi=\frac{\omega \omega_{M}}{\omega_{0}^{2}-\omega^{2}}, \omega_{0}^{2}=\frac{\gamma^{2} \Phi_{\theta \theta} \Phi_{\xi \xi}}{M^{2} \sin ^{2} \theta_{0}}, \omega_{M}=4 \pi \gamma M . \\
\Phi_{\theta \theta}=\mathrm{MH} \sin \theta_{0}-4 \pi M^{2} \cos ^{2} \theta_{0} \\
-\left|K_{1}\right|\left(-\frac{4}{3} \cos ^{4} \theta_{0}-\sin ^{4} \theta_{0}\right. \\
+7 \sin \theta_{0}^{2} \cos ^{2} \theta_{0}+\frac{10 \sqrt{2}}{3} \sin ^{3} \theta_{0} \cos \theta_{0} \\
\left.-2 \sqrt{2} \sin \theta_{0} \cos ^{3} \theta_{0}\right), \\
\Phi_{\xi \xi}=\mathrm{MH} \sin \theta_{0}-\sqrt{2}\left|K_{1}\right| \cos \theta_{0} \sin ^{3} \theta_{0} .
\end{gathered}
$$

Note that the expressions for the bulk magnetostatic waves (MSW) are obtained by replacing $q^{2}$ with $-q^{2}$ in the expressions for the surface waves (SMSW).

The solution of the equations (4)-(7) was carried out numerically, and the following values of the parameters of the YIG films were taken: saturation magnetization $4 \pi M=1760 \mathrm{Gs}$, the first constant of cubic anisotropy $K_{1} / M=-42$ Oe. The solutions of the equations graphically represented in $k_{X}-k_{Y}$ coordinates, with the frequency $\omega=2 \pi f$ as a parameter, are the isofrequency curves [23].

Figure 4 schematically shows the calculated SMSW isofrequency curves and the SMSW on SAW scattering diagram - phenomenon underlying the operation of the DMC created by SAW. Graphical calculations of the "ordinary" magnonic band gaps frequencies as functions of the angle $\beta$ between the SMSW and SAW wave vectors were performed as follows. A wave vector of SAW $Q$ was "superimposed" on a family of isofrequencies plotted in the $k_{X}-k_{Y}$ plane with a frequency interval of $5 \mathrm{MHz}$, the magnitude of which and the orientation relative to the wave vector of the incident SMSW, $k_{i}$, are known from the experiment. (The phase velocity of SAW at frequencies of $\sim 40 \mathrm{MHz}$ in the studied samples was $\left.3.3-10^{5} \mathrm{~cm} / \mathrm{s}[10]\right)$. The vector $Q$ should be positioned so that its ends lie at two isofrequencies curves that differ in frequency by the frequency of the acoustic wave, $F$ (see Figure 4). The vector $k_{r}$ is the wave vector of the reflected SMSW, and the angle $\alpha$ is its direction relative to 
the direction of the wave vector of the incident SMSW, $k_{i}$ In accordance with the theory of SMSW scattering on SAW [13] it should be

$$
\mathrm{Q}=\mathrm{k}_{\mathrm{i}}+\mathrm{k}_{\mathrm{r}} \text { and } \mathrm{f}_{\mathrm{r}}=\mathrm{f}_{\mathrm{i}}-\mathrm{F} \text {, }
$$

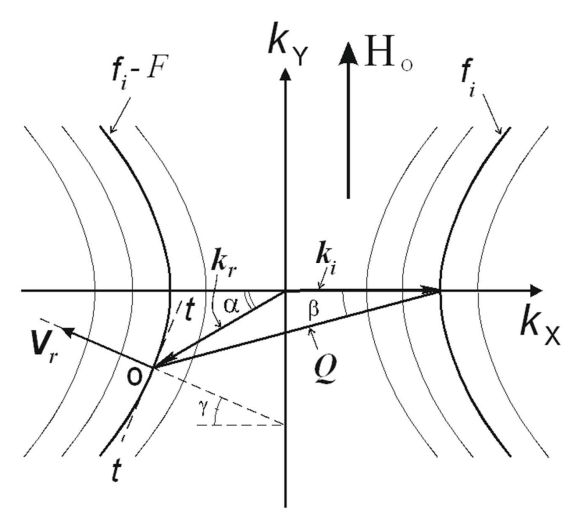

Figure 4. To calculation of isofrequency curves and SMSW propagation diagrams in SAW-DMC. Example for the "ordinary" magnonic forbidden zone.

Figure 4 shows also how the angle of the direction of the energy flow vector, $V_{r}$, (angle $\gamma$ ) in the reflected wave was graphically calculated. A tangent line ("t-t" in Figure 4) to the isofrequency curve was plotted for the frequency $f-F$ at the point of its intersection with the wave vector of the reflected wave (point "O"). The perpendicular to this tangent shows the direction of the energy flow (angle $\gamma$ ) in the reflected wave [23]. The resulting dependence of the angle $\gamma$ on the angle $\beta$ is given by the solid line 3 in Figure 3 .

The dependences of the frequencies of "ordinary" magnonic gaps calculated in this way on the angle $\beta$ between the wave vectors of SAW and the incident SMSW are shown by a solid line 1 in Figure 2 and the solid line 1 in Figure 3. Judging by Figures 2 and 3, the agreement of the experimental results with the calculated ones is satisfactory.

Similarly, the dependences of the frequencies of the "extra" magnonic gaps on the angle $\beta$ were calculated. That is, the corresponding equations for the bulk MSW were solved, families of isofrequencies curves were constructed, the vectors SAW and the incident SMSW were superimposed on it. As in the experiment, the calculation showed that the frequencies of the extra magnonic gaps lie in the initial part of the SMSW spectrum. The reflected waves turned out to be in this case bulk MSW. For the angles $\beta$ in the range of 0-15 degrees the reflected waves were the ADMSW, for angles over 15 degrees - the backward volume MSW. In order not to clutter up the presentation, we do not give here calculated isofrequency curves and SMSW -SAW vectors constructions for these cases.

The solid lines in Figure 2 represent the angular dependences of the frequencies of both the "ordinary" and "extra" magnonic bandgap zones, calculated for the sample with a YIG film thickness of 9.4 microns, observed experimentally in this sample at SAW frequency $41 \mathrm{MHz}$. The line 1 is the result for the "ordinary" magnonic gap, lines 2 and 3 are for "extra" gaps when the reflected waves are the 1-st and 2-nd modes of ADMSW, respectively. Our calculations show that such an extra gap should exist only at angles $\beta$ from 0 to $\sim 15$ degrees, but in the experiment we observed additional ("extra") gaps at any values of the angle $\beta$. We explain this by the fact that in this situation the incident SMSW can be converted to the backward volume MSW (BVMSW) when reflected. The calculations confirm this. Figure 4 shows the results of these calculations: curve 4 represents the dependence of the frequency of extramagnonic gaps on the angle $\beta$ for the case when the reflected wave is the zero mode of the BVMSW, curve 5 - when the reflected wave is the first mode of the BVMSW, and curve 6 - the second mode. Figure 4 shows that the calculation results are in satisfactory agreement with the experiment.

It should be noted that in our work, the incident MSW is not always, strictly speaking, a surface mode, since taking into account the cubic anisotropy leads to the fact that the spectrum of the true surface MSW begins not with the frequency of the ferromagnetic resonance, but somewhat higher in frequency. For the same frequency, there is a zero ADMSW mode, the dispersion dependence of which is smoothly "stitched" with the dispersion dependence of SMSW. In the experiment, the difference between the zero mode of ADMSW and SMSW does not appear. Therefore, we use the term "SMSW" everywhere in our work.

\section{Conclusion}

In this paper, we consider the propagation of SMSW in 2D geometry in a dynamic magnonic crystal created by the propagation of SAW in the YIG film - GGG substrate structure. It is shown that, as in the one-dimensional case, the DMC has magnonic bandgaps (forbidden zones) of two types ("ordinary" and "extra"). At the frequencies of the bandgap of the first type the incident SMSW undergoes reflections without transformation of the SMSW, and at the frequencies of the bandgaps of the 2-nd type reflections occurred with transformations into ADMSW at a certain range of the angles between the wave vectors of the incident SMSW and the SAW and in BBMSW at other range of this angles. The forbidden zones of the first type exist only in a certain range of angles between the wave vectors of SMSW and SAW, while the forbidden zones of the second type exist at any value of this angle. Methods are proposed and calculations of the main measured dependences are performed. A satisfactory agreement of the calculations with the experiment was obtained.

The methods and results described in this paper can be used in the development of new spin-wave devices with twodimensional geometry.

\section{References}

[1] Vasseur, J. O., Dobrzynski, L., Djafari-Rouhani, B. (1996). Magnon band structure of periodic composites. Phys. Rev. B $54,1043$. 
[2] Puszkarski, H., and Krawczyk, M. (2001). On the multiplicity of the surface boundary condition in composite materials. Phys. Lett., A 282.1, 106.

[3] Gulyaev, Yu. V., Nikitov, S. A., Zivotovskii, L. V., Klimov, A A., Tailhades, Ph., Presmanes, Bonningue, L. C., Tsai, T. S., Vysotskii, S. L., Filimonov. Y. A. (2003). Ferromagnetic films with magnon bandgap periodic structures: Magnon crystals. JETP Lett. 77, 567.

[4] Chumak, A. V., Vasyuchka, V. I., Serga, A. A., Kostylev, M. P., Tiberkevich, V. S., Hillebrands, B. (2012). StorageRecovery Phenomenon in Magnonic Crystal. Phys. Rev. Lett. 108, 257207.

[5] Gallardo, R. A., Schneider, T., Roldán-Molina, A., Langer, M., Fassbender, J., Lenz, K., Lindner, J., Landeros, P. (2018). Dipolar interaction induced band gaps and flat modes in surface-modulated magnonic crystals. Phys. Rev. B. V. 97 (14), 144405.

[6] Chumak, A. V., Serga, A. A., Hillebrands, B. (2017). Magnonic crystals for data processing. J. Phys. D: Appl. Phys. 50 (24), 244001.

[7] Sadovnikov, A. V., Odintsov, S. A., Beginin, E. N., Grachev, A. A., Gubanov, V. A., Sheshukova, S. E., Sharaevskii, Yu. P. \& Nikitov, S. A. (2018). Nonlinear Spin Wave Effects in the System of Lateral Magnonic Structures. Jetp Lett. 107, 25-29.

[8] Kryshtal R. G., Kundin A. P., Medved A. V. (2019). A microwave nonreciprocal notch filter tunable by a surface acoustic wave in dynamic magnonic crystals. Instruments and Experimental Techniques. 62 (1), 42-46.

[9] Gubanov, V. A., Sheshukova, S. E., Nikitov, S. A. Sadovnikov, A. V. (2021). Multimode unidirectional spinwave coupling in an array of non-identical magnonic crystals near band gap frequencies. J. Phys. D: Appl. Phys. 54, 245001

[10] Kryshtal R. G., Medved A. V. (2012). Surface acoustic wave in yttrium iron garnet as tunable magnonic crystals for sensors and signal processing applications. Appl. Phys. Lett. V. 100 (19), 192410

[11] Mednikov, A. M., Popkov, A. F., Anisimkin, V. I., Nam, B. P., Petrov, A. A., Spivakov, A. A., and Khe, A. S. (1981). Inelastic scattering of a surface spin wave from a surface acoustic wave in a thin, iron-yttrium-garnet film. JETP Lett. $33(12), 632$.

[12] Popkov, A. F. (1981). The dispersion of surface magnetostatic waves in the presence of a traveling wave of elastic deformation. Mikroelectronika. 10, 446.

[13] Popkov, A. F. (1985). Difraction of Spin-waves (Magnetostatical) on the Acoustical Wave. Fiz. Met. Metalloved. 59, 463.

[14] Gulyaev, Yu. V., Krishtal, R. G., Medved, A. V., Sorokin, V. G. (1986). Nonelastic scattering of surface magnetostatic waves on the surface acoustic-wave in the monolythic $\mathrm{ZnO}$ IYG-GGG structure. Pis'ma Zh. Tekh. Fiz. 12, 502.

[15] Kryshtal, R. G., Medved, A. V., Nikitin, I. P., and Drobiazko, I. B. (1986). Anisotropy-caused Nonelastic Scattering of Surface Magnetostatic Waves on an Acoustic Wave of YIG Films. Zh. Tech. Fiz. 56, 1835.
[16] Kryshtal, R. G., Medved, A. V., and Popkov, A. F. (1994). Effect of Decay of Surface Magnetostatic Waves on the Parameters of Their Scattering by a Surface Acoustic Wave in Yttrium Iron Garnet Films. J. Commun. Technol. Electron. 39, 132.

[17] Hanna, S. M., Murphy, G. P., Sabetfakhri, K., and Stratakis, K. (1990). Experimental investigation of scattering of magnetostatic waves by surface acoustic waves. Proc. IEEE Ultrason. Symp. 1, 209.

[18] Kryshtal, R. G., Medved, A. V. (2019). Dynamic magnonic crystals for measurements of parameters of surface spin waves in yttrium-iron garnet films. JMMM. 491, 165599.

[19] Kryshtal, R. G., Medved, A. V. (2021). Dynamic Magnonic Crystals for Measuring the Dispersion of Bulk Magnetostatic Spin Waves Caused by Magnetic Anisotropy in YIG Films. Instruments and Experimental Techniques. 64 (1), 121-126.

[20] Kryshtal, R. G., Medved, A. V. (2019). Surface acoustic waves in dynamic magnonic crystals for microwave signals processing. Ultrasonics. 94, 60-64.

[21] Kryshtal, R. G., Kundin, A. P., Medved, A. V. (2019). A microwave nonreciprocal notch filter tunable by a surface acoustic wave in dynamic magnonic crystals. Instruments and Experimental Techniques. 62 (1), 42-46.

[22] Kryshtal, R. G., Medved, A. V. (2015). Nonreciprocity of spin waves in magnonic crystals created by surface acoustic waves in structures with yttrium iron garnet. JMMM. 395, 180-184.

[23] Lock, E. H. (2008). The properties of isofrequency dependences and the laws of geometrical optics. PhysicsUspekhi. 51 (4), 375-393.

[24] Krishtal, R. G., Medved, A. V. (1987). Dispersion of magnetostatic waves, induced by anisotropy in ironyttrium-garnet films. Zhurnal Tekhnicheskoi Fiziki, 57, 1936-1941.

[25] Voronenko, A. V., Gerus, S. V. (1986). Diffraction of surface magnetostatic waves on magnetic lattices in the Raman-Nath regime. Pisma v Zhurnal Tekhnicheskoi Fiziki, 12 (10), 632635 .

[26] Krishtal, R. G., Medved, A. V., Osipenko, V. A., Shakhnazarian, D. G., (1986). Noncolinear inelastic-scattering of superficial magnetostatic waves on the superficial acousticwave. Pis'ma v Zhurnal Tekhnicheskoi Fiziki. 12 (12), 14481451.

[27] Kryshtal, R. G., Medved, A. V. (2017). Nonlinear spin waves in dynamic magnonic crystals created by surface acoustic waves in yttrium iron garnet films. J. Phys. D: Appl. Phys. 50, 495004.

[28] Kryshtal, R. G., Medved, A. V. (2017). Influence of magnetic anisotropy on dynamic magnonic crystals created by surface acoustic waves in yttrium iron garnet films. JMMM. 426, 666669.

[29] Damon R W., Eshbach, J. R. J., (1961). Magnetostatic modes of a ferromagnet slab. Phys. Chem. Solids, 19 (3-4), 308-320.

[30] Lax N. and Batton K. J., (1962). Microwave Ferrites and Ferrimagnetics (New York: McGraw-Hill). 